\title{
The isotropic-nematic phase transition in hard, slightly curved, lens-like particles
}

\author{
Giorgio Cinacchi ${ }^{1, \text { a) }}$ and Alessandro Tani ${ }^{2}$ \\ ${ }^{1}$ Departamento de Física Teórica de la Materia Condensada and Instituto de Física de la Materia \\ Condensada, Universidad Autónoma de Madrid, Campus de Cantoblanco, E-28049 Madrid, Spain \\ ${ }^{2}$ Dipartimento di Chimica, Università di Pisa, Via Risorgimento 35, I-56100 Pisa, Italy
}

(Received 14 July 2014; accepted 30 September 2014; published online 17 October 2014)

\begin{abstract}
Monte Carlo numerical simulations are used to study in detail how the characteristics of the isotropicnematic phase transition change as infinitely thin hard platelets are bent into shallow lens-like particles. First, this phase transition in the former reference model system is re-examined and more accurately located. Then, it is shown quantitatively that this already quite weak but distinctly firstorder phase transition weakens further upon curving the platelets to such an extent that, thanks to the thinness of these particles that does not favor its pre-emptying by a transition to a (partially) positionally ordered phase, an isotropic-nematic tricritical point limit can be arbitrarily closely approached. C 2014 AIP Publishing LLC. [http://dx.doi.org/10.1063/1.4897565]
\end{abstract}

\section{INTRODUCTION}

The isotropic (I) - nematic (N) liquid-crystal ${ }^{1}$ phase transition has been much studied ${ }^{1-3}$ and a consensus exists on its character: it is a weakly first-order phase transition, in that there is a discontinuity in at least one of the free energy first derivatives and that discontinuity is quite small. The value of the nematic order parameter ${ }^{1}\left(S_{2}\right)$ at the phase transition is not that small though, being found typically in the range $0.3-0.4{ }^{1}$ The weakness of the phase transition manifests itself in the presence, within the I phase, of significant pre-transitional effects. ${ }^{1}$ The latter are reminiscent of the approaching to a second-order phase transition and are generally interpreted in terms of the phenomenological Landau-de Gennes theory. ${ }^{1,4,5}$ Consensus on the critical character of this phase transition ${ }^{6}$ is not as firm. Most experimental data point to it being mean-field tricritical (e.g., Refs. 7 and 8) but not definitively: the main difficulty being that the innermost critical region is made unaccessible by the intervening two-phase coexistence.

To approach, ideally as closely as wished, this critical region would be interesting but does not appear to be an easy task. One would require a physical system in which the IN phase transition shares two, not necessarily synergic, features. On tuning certain relevant thermodynamic and/or structural parameters, the already weakly first-order IN phase transition must progressively weaken further and not be pre-emptied by a transition of the I phase to a (partially) positionally ordered phase.

In terms of the elementary models put forward over the years to study liquid-crystalline phase behavior and properties (e.g., Ref. 9), the simplest, yet realistic, models one's attention is turned to are freely translating and rotating non-spherical particles of the hard-body type. They possess the essential pre-requisite of the anisotropic shape, being short-range re-

a)Electronic mail: giorgio.cinacchi@uam.es pulsive interactions known to be responsible for the structure of a fluid. ${ }^{10}$ That they also provide a good representation of real colloidal particles, whose synthesis has much progressed in these years, ${ }^{11}$ adds to the interest in them.

For a hard-body particle system, temperature plays no role in shaping its phase behavior, so one is left with modifying the geometry of the model in order to find one in which the IN phase transition weakens, ideally at will, without being overcome by the intervention of a partially positionally ordered phase.

In order not to favor such an ordered phase, a model particle with an extreme value of the aspect ratio is to be sought. Out of the two general classes in which mesogenic particles have been sub-divided, ${ }^{1}$ calamitic, or rod-like, and discotic, or disc-like, the latter seems more suitable. In fact, Onsager (second-virial) theory, ${ }^{12}$ exact for long and thin hard rods, shows that the IN phase transition is actually strongly firstorder in that case. The discoid analogue of a long and thin hard rod is the infinitely thin hard platelet.

This basic statistical-mechanical model was introduced and studied, by Monte Carlo (MC) numerical simulation, ${ }^{13-15}$ in Ref. 16. Focus of that study was indeed the IN phase transition that occurs in a system of these particles. This phase transition was found to be weakly first-order and, expectedly, unsatisfactorily described by a second-virial theory. The IN phase transition in hard platelets was re-examined in Ref. 17. More recent studies include the formulation of a density functional theory (DFT) that improves upon Onsager theory results for coexistence densities: ${ }^{18}$ usage of this DFT along with MC simulations to study properties of inhomogeneous hard platelet fluids $;{ }^{19}$ a very detailed examination of the I phase equation of state and structure, ${ }^{20}$ where a range of integral equation theories along with the above-mentioned DFT were thoroughly tested; the calculation of elastic constants ${ }^{21}$ in the $\mathrm{N}$ phase.

Two recent studies ${ }^{22,23}$ showed that, on curving hard platelets into hard partial spherical surfaces or spherical cap 
particles (having a shape reminding that of a contact lens, if little or moderately curved, or that of a bowl or vase, otherwise), the IN phase transition competes with a tendency of these particles to aggregate. This tendency, an example of geometric frustration, ${ }^{24}$ leads to a weakening of the IN phase transition until the $\mathrm{N}$ phase becomes unstable and is superseded by a cluster I phase; from this a cluster columnar phase develops at higher densities. While these two previous works focused on such cluster phases, in this work a closer attention is paid to the first process. More MC numerical simulations are carried out to study how the IN phase transition features change upon curving platelets slightly into lens-like particles.

\section{MODELS AND COMPUTATIONAL DETAILS}

Particles belonging to the hard spherical cap class can be identified by a single parameter $R^{*}=R / \sigma, R$ being the radius of the sphere from which the cap has been excised and $\sigma$ is the unit of length, defined in such a way that $\sigma^{2}$ is the area of the cap's two-dimensional surface (Fig. 1).

Being a reference system, extensive new MC calculations were carried out for hard platelets, corresponding to $R^{*}$ $\rightarrow \infty$, along with MC calculations for hard spherical caps with $R^{*}=2$ and $8 / 5$. It was decided to carry out the MC calculations in the canonical $(N V T)^{13,15}$ and isobaric(-isothermal) $(N P T)^{14,15}$ ensembles, as done in Ref. 16, rather than in the Gibbs or grand-canonical $(\mu V T)^{15}$ ensembles, as done in Ref. 17. Usually standard cuboidal (but occasionally also truncated octahedral) periodic boundary conditions ${ }^{15}$ were implemented. Previous calculations ${ }^{22,23}$ found that for $R^{*}=3 / 2$ the $\mathrm{N}$ phase was stable only for systems with a number of particles $N \simeq 500$, while for $R^{*}=1$ not even a system with $N \simeq 100$ was able to stabilize a $\mathrm{N}$ phase. Thus, several system sizes were investigated, with $N$ ranging from $\simeq 500$ to $\simeq 11000$, with a few calculations for hard platelets performed with $N=64000$. Most of the MC-NPT runs were carried out with 4000 or 5000 particles, while $N$ in the MC- $N V T$ runs was 8000 . In the calculations on hard platelet systems, the specific overlap criterion for these particles ${ }^{16}$ was used, while the more general overlap criterion for hard spherical caps $^{22,23,26}$ was employed in the other two cases. That the latter recovers the former overlap criterion in the limit $R^{*} \rightarrow \infty$ was checked previously. ${ }^{22}$

Every MC calculation was organized in cycles, each one, on average, consisting of: (a) $N$ attempted translational moves

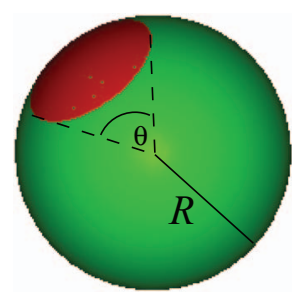

FIG. 1. Illustration of a spherical cap (red or darker gray) together with its parent sphere (green or lighter gray) of radius $R$; the area of the spherical cap surface is equal to $\sigma^{2}$; once set $R^{*}=R / \sigma$, the angle $\theta$ is such that $\cos \frac{\theta}{2}$ $=1-1 /\left(2 \pi R^{* 2}\right)$. This image was created with the program QMGA. ${ }^{25}$ to change the position $\mathbf{r}_{i}$ of the pole of a particle $i$; (b) $N$ attempted rotational moves to change the orientation of a particle $i$, its orientation being described by the unit vector $\widehat{\mathbf{u}}_{i}$ lying along the joint of the center of the parent sphere, whose position is $\mathbf{X}_{i}$, with the pole; these rotational moves were performed either with the method described in Ref. 16 or the usual Barker-Watts method; ${ }^{15}$ (c) an attempt to flip a particle while keeping the position of its pole; (d) in the MC-NPT calculations, an attempt to change shape and volume of the computational box. (For hard platelets, vector $\mathbf{r}$ ends up to describe the position of a platelet center and $\widehat{\mathbf{u}}$ is aligned along a platelet normal passing through its center, while a flip is non-influential). For each type of move, its maximum size was adjusted so that the corresponding move had an acceptance rate of $\sim 30 \%$. If needed, maximum sizes were suitably modified to approach the above percentage before starting a calculation but never during it not to violate the detailed balance condition. The choice of which move to attempt as well as, in the cases (a), (b), and (c), that of which particle to move were made randomly. In nearly all the calculations, the random number generator used was mt $19937 .^{27}$

Equilibration runs were started either from a low density lattice configuration having all particles perfectly aligned or an equilibrated configuration obtained at a nearby state point or by replicating twice along each direction a previously equilibrated configuration at the same state point. Equilibration runs lasted $10^{6} \mathrm{MC}$ cycles at least and were followed by production runs of as many MC cycles. During these production runs, a number of quantities were calculated: density, $\rho$, measured in units of $\sigma^{-3}$, in the isobaric MC runs; pressure, $P$, measured in units of $k_{B} T \sigma^{-3}$, with $k_{B}$ the Boltzmann constant and $T$ the temperature, and evaluated following the method described in Ref. 16, in the canonical MC runs; the nematic order parameter, $S_{2}$, calculated according to the standard procedure ${ }^{28}$ in any $\mathrm{MC}$ run, this calculation providing also the instantaneous nematic director $\widehat{\mathbf{n}}$, from which the polar order parameter $S_{1}=\langle\widehat{\mathbf{u}} \cdot \widehat{\mathbf{n}}\rangle$, with \langle\rangle meaning an average over particles and configurations, can in turn be evaluated; several positional and orientational correlation functions. Uncertainties were estimated either using the blockaverage $\operatorname{method}^{29}$ or running a few independent runs and evaluating the respective mean and standard deviation.

For the three model particles investigated, the series of MC calculations were organized in the following way. The MC-NPT method was first used to trace the equation of state, checking the stability of the $\mathrm{N}$ phase on going to larger systems and bracketing accurately the value of thermodynamic quantities at the IN phase transition. Then, the MC- $N V T$ method was used especially in the close neighborhood of and within the IN coexistence region to improve the accuracy of the phase transition location. In determining approximate values for the thermodynamic quantities at coexistence, effort was spent to observe a Mayer-Wood loop ${ }^{30,31}$ within the coexistence region; pressure, $P_{\mathrm{IN}}$, and densities, $\rho_{\mathrm{l}}$ and $\rho_{\mathrm{N}}$, at coexistence were estimated by applying an equal area construction. This also led to an estimate of the interfacial free energy per particle, ${ }^{31} \Delta$ f. Statistical errors on these quantities were in turn estimated from the errors bars on the raw equation of state data. 


\section{RESULTS AND DISCUSSION}

\section{A. Thermodynamics}

Fig. 2 shows the equation of state for the three models investigated. The respective insets zoom in on the IN coexistence region. For the case with $R^{*}=8 / 5$, error bars on the values of $P$ calculated in the MC- $N V T$ simulations are also drawn; in the other two cases, they are of a similar size. In all the three cases, a Mayer-Wood loop can be discerned. Table I provides, for the three models, the estimated value of pressure and the densities at coexistence as well as that of the interfacial free energy.

Focusing on the equation of state and IN coexistence data for hard platelets, a good agreement is noted ${ }^{32}$ with the past results of Ref. 16; significant differences are seen only in the neighborhood of the phase transition. In that work, using mostly systems with $N=100$ and Widom particle insertion method and thermodynamic integration ${ }^{15}$ calculations, pressure and densities at coexistence were estimated to be, respectively, 10.30 and 2.81 and 2.87. While these numbers compare favorably with those of Table I, one observes that in the latter case pressure and $\mathrm{N}$ phase density at coexistence have shifted to a larger value, owing to the larger size of the systems investigated here. Present data agree well also with the recent $\mathrm{MC}$ results reported for the IN phase transition for thin hard cut-spheres using systems with $N=288$ and $2048 .{ }^{33}$ Data of Ref. 33 and present data are instead not consistent with the IN coexistence data of Ref. 17. Those works report that systems with $N$ in the range 400-2200 were used and, by employing MC methods that rely on particle insertions, they found $\rho_{\mathrm{l}} \sigma^{3}$ $=2.56 \pm 0.01$ and $\rho_{\mathrm{N}} \sigma^{3}=2.77 \pm 0.03$. Here, systems at $\rho \sigma^{3}$ $=2.77 \pm 0.03$ are invariably found to be in the I phase.

On decreasing $R^{*}$, the IN shifts to higher densities and pressure and the width of the coexisting region, $\Delta \rho_{\mathrm{IN}}=2\left(\rho_{N}-\rho_{I}\right) /\left(\rho_{N}+\rho_{I}\right)$, shrinks from $\simeq 6.77 \%$ for hard platelets, to $\simeq 3.15 \%$ for hard spherical caps with $R^{*}=2$, to $\simeq 1.80 \%$ for hard spherical caps with $R^{*}=8 / 5$.

Concomitantly, the interfacial free energy per particle diminishes while reducing $R^{*}$. The value of $\Delta \mathrm{f}$ for hard platelets is consistent with the trend that the IN free energy barrier in thin hard cut-spheres exhibits with the aspect ratio as determined in Ref. 33(a) using an umbrella sampling MC technique. From the value of $\Delta \mathrm{f}$ one can arrive at a gross estimate of the interfacial tension, $\gamma \sim \Delta \mathbf{f} \bar{\rho} L$, with $\bar{\rho}=\left(\rho_{I}+\rho_{N}\right) / 2$ and $L^{3}$ the system volume. In this way a value of $\gamma \sigma^{2} / k_{B} T$ $\sim 7.3 \times 10^{-3}$ is obtained for hard platelets. This number compares fairly well with previous estimates for $\gamma$ obtained from DFT and dedicated numerical simulations ${ }^{19(a)}$ as well as with experiments on colloidal disc suspensions. ${ }^{34}$ On going to hard spherical caps with $R^{*}=2$, the estimate for $\gamma \sigma^{2} / k_{B} T$ turns out to be $5.8 \times 10^{-3}$, while for the hard spherical caps with $R^{*}=8 / 5$ it drops to $3.3 \times 10^{-3}$.

\section{B. Structure factor}

One complementary indication of the phase transition weakening on going to smaller values of $R^{*}$ is provided by the value that the structure factor $S$ takes on at the minimum value $k_{\min }$ of the modulus of the wave-vectors $\mathbf{k}$ compatible
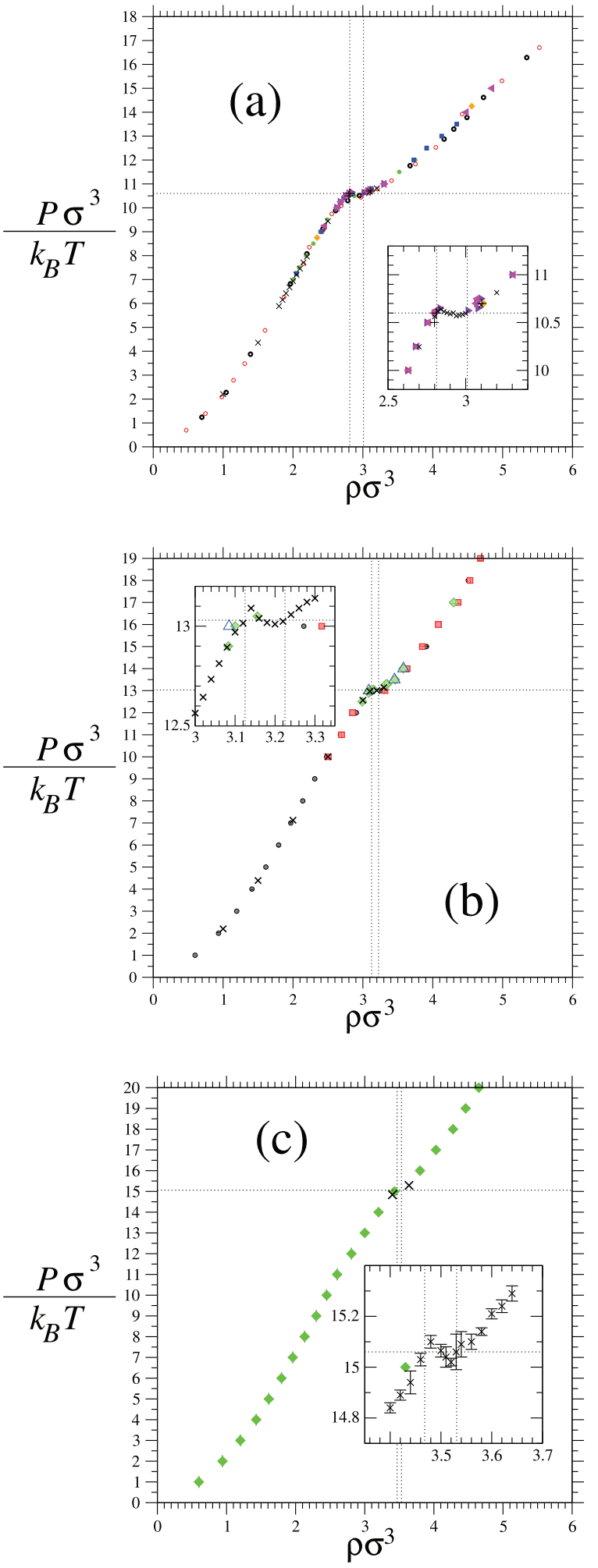

FIG. 2. (a) Equation of state for hard platelets, $R^{*} \rightarrow \infty$, obtained using the MC-NPT method with $N=541$ (green full circles), $N=1373$ (blue full squares), $N=2137$ (orange full diamonds), $N=4000$ (either left-magenta or right-violet full triangles), $N=10984$ (black asterisks) or the MC- $N V T$ method with $N=8000$ (black crosses). Empty black or red little circles are data taken from Ref. 16. (b) Equation of state for hard spherical caps with $R^{*}$ $=2$ obtained using the MC-NPT method with $N=1013$ (gray full circles), $N$ $=2000$ (red full squares), $N=4096$ (green full diamonds), $N=8101$ (blue empty triangles) or the MC- $N V T$ method with $N=8000$ (black crosses). (c) Equation of state for hard spherical caps with $R^{*}=8 / 5$ obtained using the MC-NPT method with $N=5000$ (green full diamonds) or the MC- $N V T$ method with $N=8000$ (black crosses). 
TABLE I. Pressure and densities at the IN coexistence and interfacial free energy for the three models investigated.

\begin{tabular}{lcccc}
\hline \hline$R^{*}$ & $P_{\mathrm{IN}} \sigma^{3} / k_{B} T$ & $\rho_{\mathrm{l}} \sigma^{3}$ & $\rho_{\mathrm{N}} \sigma^{3}$ & $10^{4} \Delta \mathrm{f} / k_{B} T$ \\
\hline$\infty$ & $10.600 \pm 0.005$ & $2.813 \pm 0.004$ & $3.010 \pm 0.004$ & $1.8 \pm 0.2$ \\
2 & $13.03 \pm 0.03$ & $3.125 \pm 0.002$ & $3.225 \pm 0.01$ & $1.35 \pm 0.15$ \\
$8 / 5$ & $15.06 \pm 0.03$ & $3.468 \pm 0.002$ & $3.531 \pm 0.007$ & $0.725 \pm 0.15$ \\
\hline \hline
\end{tabular}

with the length $L$ of the computational box edges in the MC$N V T$ simulations. The structure factor was calculated as

$$
S(k)=\frac{1}{N}\left\langle\sum_{l=1}^{N} \mathrm{e}^{-i \mathbf{k} \cdot \mathbf{r}_{l}} \sum_{m=1}^{N} \mathrm{e}^{i \mathbf{k} \cdot \mathbf{r}_{m}}\right\rangle,
$$

with \langle\rangle indicating an average over configurations and the elements of that set of wave-vectors $\mathbf{k}$ of modulus $k=|\mathbf{k}|$ compatible with the computational box. Thus, it is the isotropic structure factor that was calculated, both in the I and $\mathrm{N}$ phase, linked to the pair correlation function, $g(r)$, via the relationship ${ }^{10}$

$$
S(k)=1+4 \pi \rho \int_{0}^{\infty} d r r^{2}[g(r)-1] \frac{\sin k r}{k r},
$$

$r$ being the distance separating the poles of two particles. The value of $S\left(k_{\min }\right)$ has a bearing on the (isothermal) compressibility $\kappa_{T}$ in that $\lim _{k \rightarrow 0} S(k)=S(0)=k_{B} T \rho \kappa_{T}$. On approaching the phase transition from the I side, $S\left(k_{\min }\right)$ first increases, then reaches a maximum within the coexistence region and finally decreases. This behavior, shown in Fig. 3, is expected in a finite system that, in the thermodynamic limit, will exhibit a first-order phase transition: in that limit, $\kappa_{T}$ increases on approaching the phase transition from either side, its further rise being then interrupted by the setting in of the two-phase coexistence. Rather than the precise shape of the curve and value of the numbers, what is more noteworthy in this figure is the

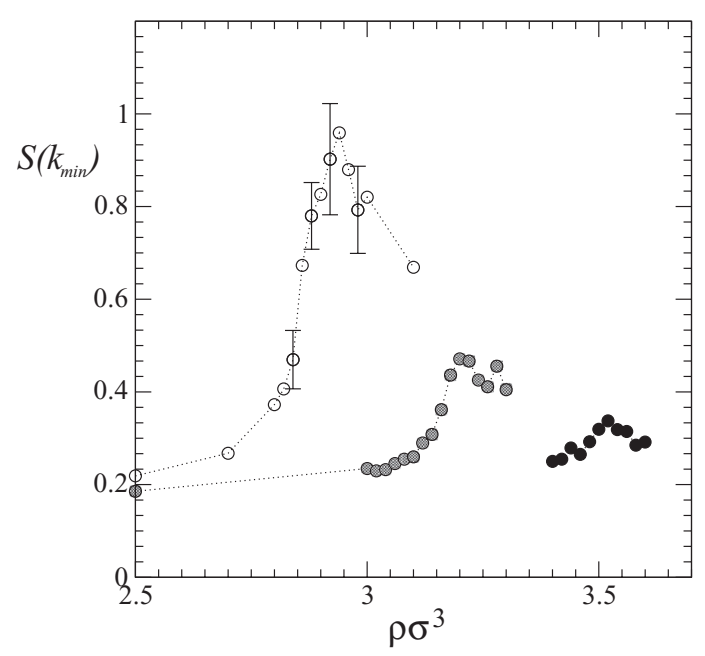

FIG. 3. Value of the structure factor at the smallest possible value of the wave-vector modulus, $S\left(k_{\min }\right)$, as a function of density, $\rho$, calculated in the MC- $N V T$ simulations. Results shown are for hard platelets, $R^{*} \rightarrow \infty$ (white circles), hard spherical caps with $R^{*}=2$ (gray circles) and hard spherical caps with $R^{*}=8 / 5$ (black circles). Error bars on $S\left(k_{\text {min }}\right)$ are shown for the hard platelet case at certain values of $\rho$; these bars are representative of the error size that affects these data for all the systems investigated. damping that the upswing of $S\left(k_{\min }\right)$ experiences on increasing the particle curvature. This indicates that, as particles are bent, the IN phase transition occurs with progressively smaller long-range density fluctuations.

\section{Orientational properties}

While both equation of state and structure factor data are consistent with a weakening of the first-order character of the phase transition, the nematic order parameter $S_{2}$ shows the same trend with density for the three systems investigated. $S_{2}$ keeps being characterized by the usual abrupt rise at the phase transition, just shifted to larger values of density as $R^{*}$ decreases [Fig. 4(a)]. The similarity in the density dependence of $S_{2}$ in the vicinity of the phase transition for the three models prompts an attempt to make these data coalesce on a single curve.

Once density is replaced by temperature as independent variable, this attempt complies with the many evidences that the thermal behavior of experimental $S_{2}$ data in thermotropic nematic liquid crystals shows corresponding-state-like features (e.g., Ref. 35). Usually, experimental $S_{2}$ data are supposed to vary with $T$ according to the following function

$$
S_{2}(T)=S_{2}^{*}+c\left(1-\frac{T}{T^{*}}\right)^{\beta}, T<T^{*},
$$

with $\beta$ a pseudo-critical exponent, $T^{*}$ a temperature slightly higher than the IN phase transition temperature, $c$ a constant, and $S_{2}^{*}$ the value of $S_{2}$ at $T^{*}$. If the latter is set equal to 0 , Haller expression ${ }^{36}$ is recovered; a non-zero value of $S_{2}^{*}$ was introduced ${ }^{37}$ to improve the fit of certain experimental data. If Haller expression ${ }^{36}$ is used, $\beta$ turns out to be 0.2 or less for a number of compounds. ${ }^{38}$ If the constraint of $S_{2}^{*}=0$ is released, fit over $S_{2}$ data for alkyl-cyano-biphenyl nematogens produced $S_{2}^{*}=0.14 \pm 0.05$ and $\beta=0.24 \pm 0.01 .{ }^{37}$ Results such as this are taken as the most convincing indication for a mean-field tricritical nature of the IN phase transition, being $1 / 4$ the value predicted by a mean-field theory for exponent $\beta$ at a tricritical point. ${ }^{1}$ The fact that allowing for a value of $S_{2}^{*}>0$ leads to an increase of $\beta$ may just be resulting from the attempt, by the fitting function, to capture the abrupt rise of $S_{2}$ in the vicinity of the phase transition: if a fraction of this rise is already accounted for by a finite value of $S_{2}^{*}$, clearly a smaller value of $\beta$ is demanded for achieving the goal.

For an athermal system like those considered here, Eq. (3) translates into

$$
S_{2}(\rho)=S_{2}^{*}+c\left(\frac{\rho}{\rho^{*}}-1\right)^{\beta}, \rho>\rho^{*},
$$

with $\rho^{*}$ a density slightly smaller than $\rho_{\mathrm{N}}$. The three parameters, $\beta, \rho^{*}$, and $S_{2}^{*}$ appear to be highly correlated to one another. In order to partly alleviate this problem it was decided to scale the value of the density by that value of density, among those investigated, at which $S_{2}$ first took a value larger than 0.3 . In this way, the data for the three models were all described in terms of the scaled density $\varepsilon=\left(\frac{\rho}{\rho^{*}}-1\right)$ and appear, within statistical errors, to lie on a common curve, at least in the vicinity of $\varepsilon=0$ [Fig. 4(b)]. If a fit with Eq. (4) is limited to the steeply ascending portion of the curve 

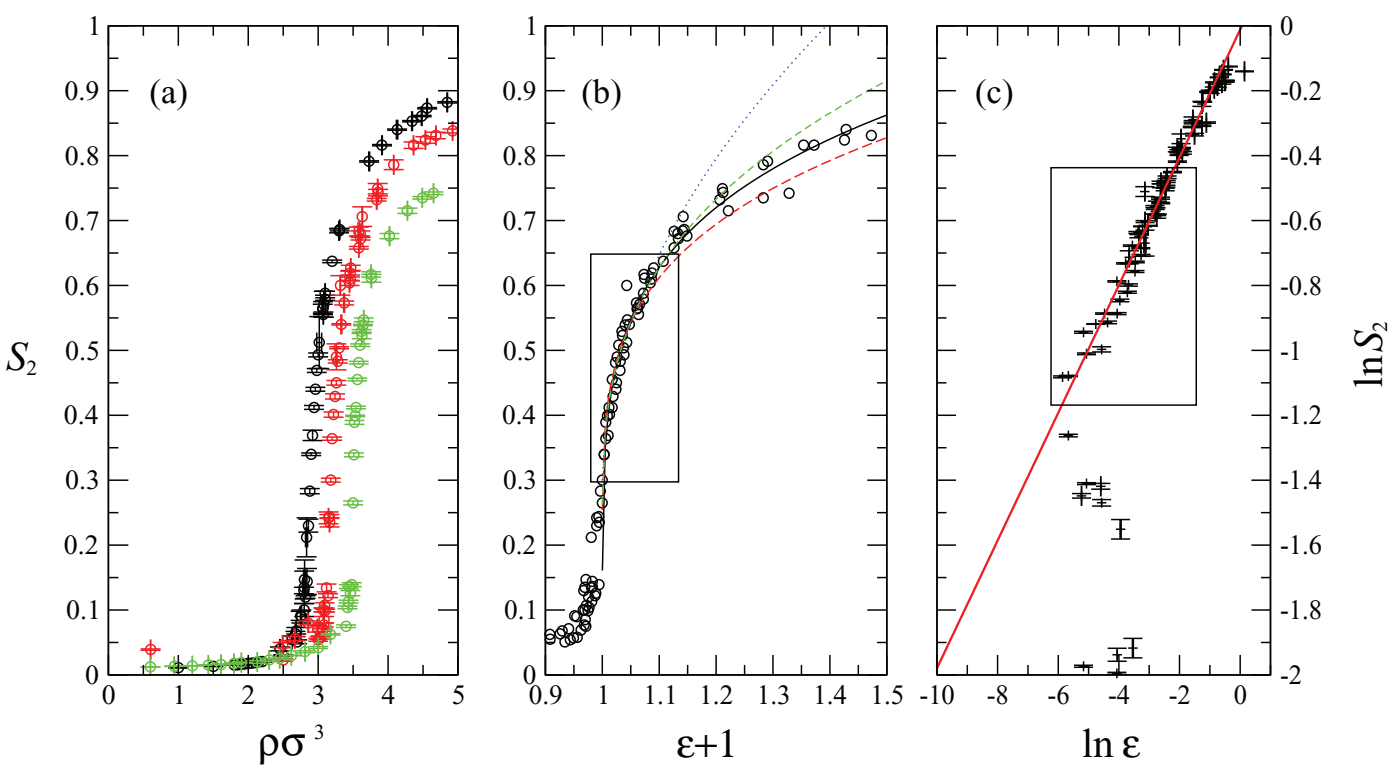

FIG. 4. (a) Nematic order parameter, $S_{2}$, as a function of density, $\rho$, for $R^{*} \rightarrow \infty$ (black leftward empty circles), $R^{*}=2$ (red central empty circles) and $R^{*}=8 / 5$ (green rightward empty circles). (b) The same data (empty circles) plotted in terms of $\varepsilon+1$, together with the curves obtained via fitting to Eq. (4) with $\beta=1 / 2$ (blue dotted curve), 1/3 (green dashed curve), and 1/4 (red dashed curve), all having a value for $S^{*}>0$, and the curve obtained via fitting to Eq. (4) and taking $S^{*}=0$ and resulting to have $\beta=0.197$ (black solid curve). (c) The same data as in (b) (black crosses) on a logarithmic scale together with the curve obtained via fitting to Eq. (4) and taking $S^{*}=0$ and resulting to have $\beta=0.197$ (red solid curve).

in the vicinity of $\varepsilon=0$, specifically to those values of $S_{2}$ in the range $(0.3 ; 0.65)$ [enclosed in the rectangle shown in Fig. 4(b)], good and, within statistical errors, essentially equivalent reproductions of the data can be obtained by adjusting $S_{2}^{*}$ and $\beta$. Fig. 4(b) shows the analytic curves obtained by setting $\beta=1 / 2,1 / 3$, and $1 / 4$. The corresponding optimized values of $S_{2}^{*}$ obtained were, respectively, $0.28,0.22$, and 0.18 : clearly, the larger is the value of $S_{2}$ at which one is starting from, the larger is the value of $\beta$ required to track closely the nematic order parameter data. The performance of the various analytic curves starts differentiating from one another if one requires that the analytic fitting function reproduces the trend of the $S_{2}$ also for larger values of $\varepsilon$. Under this "policy," a value of $\beta=1 / 2$ turns out unsatisfactory, while a value of $\beta=1 / 4$ corresponds to a fit of a quality that outperforms only slightly that of a fit with $\beta=1 / 3$. The best fit is nonetheless achieved by a Haller function with $\beta=0.197 \pm 0.006$. This fit, again limited to values of $S_{2}$ within the rectangle of Fig. 4(b), is able to satisfactorily reproduce the joint values of $S_{2}$ for the three models up to $\varepsilon=0.5$, as also Fig. 4(c) shows. This optimized value of $\beta$ is in agreement with those from similar analyses of a number of experimental data ${ }^{38}$ and of past and more recent numerical simulations carried out with either idealized or atomistic thermotropic liquid crystal models. ${ }^{39}$ While certainly not far away from the mean-field prediction for $\beta$ at a tricritical point, this value, taken in isolation, cannot objectively be used in full support of a mean-field tricritical character of the IN phase transition.

Information on the value of $S_{2}$ is also contained in the tail of the second-rank pair orientational correlation function, $g_{2}(r)$. Functions of this type are defined as

$$
g_{n}(r)=\left\langle\frac{\sum_{i=1}^{N} \sum_{j \neq i}^{N} \delta\left(r-r_{i j}\right) P_{n}\left(\hat{\mathbf{u}}_{i} \cdot \hat{\mathbf{u}}_{j}\right)}{\sum_{i=1}^{N} \sum_{j \neq i}^{N} \delta\left(r-r_{i j}\right)}\right\rangle,
$$

with $\delta()$ the usual $\delta$-function, $r_{i j}$ the distance separating the poles of two particles $i$ and $j, P_{n}()$ the $n$ th-rank Legendre polynomial, and \langle\rangle meaning a statistical average over configurations. The functions $g_{n}(r)$ are such that: $\lim _{r \rightarrow \infty} g_{n}(r)$ $=S_{n}^{2}$, with $S_{n}$ the mean $n$ th-rank Legendre polynomial. In particular $\lim _{r \rightarrow \infty} g_{2}(r)=S_{2}^{2}$. This relationship is accurately obeyed in the $\mathrm{N}$ phase, where it offers another way of determining the value of $S_{2}$. It is also obeyed in the I phase but here the finiteness of the system size prevents $S_{2}$ going to 0 . This is a well-known finite size effect, ${ }^{16(b)}$ here appreciable in Fig. 4(a). While it does not prejudice the identification of the phase as I or $\mathrm{N}$ sufficiently far away from the phase transition, on approaching the latter from the I phase side, it affects negatively the analysis of the long-range limit of $g_{2}(r)$. In the I phase, the tail of $g_{2}(r)$ contains information on the orientational correlation length, $\xi$, as one has: $\lim _{r \rightarrow \infty} g_{2}(r)$ $\sim r^{-1} \exp (-r / \xi)$. In order to extract a value for $\xi$ as unambiguous as possible, the system should be of so large a size to allow for sampling large enough $r$ 's at which the above limiting relationship holds yet not so large that the value of $g_{2}(r)$ becomes comparable to $S_{2}^{2}$. Strict adhering to both of these conditions has the inevitable consequence that the interval of analyzable $r$ 's shrinks considerably. One way to partly alleviate this problem is to fit the whole tail of $g_{2}(r)$ to the following function:

$$
g_{2}^{f i t}(r)=S_{2}^{2}+c \frac{\mathrm{e}^{-r / \xi}}{r},
$$

with $S_{2}$ treated as a fitting parameter and $c$ an additional fitting parameter. By proceeding in this way, the values of $\xi$ reported in Fig. 5 as a function of density were obtained for the three models. It would be of importance to arrive at a knowledge on how quantitatively $\xi$ increases with density as this would inform on the critical nature of the IN phase transition. The $\xi$ 


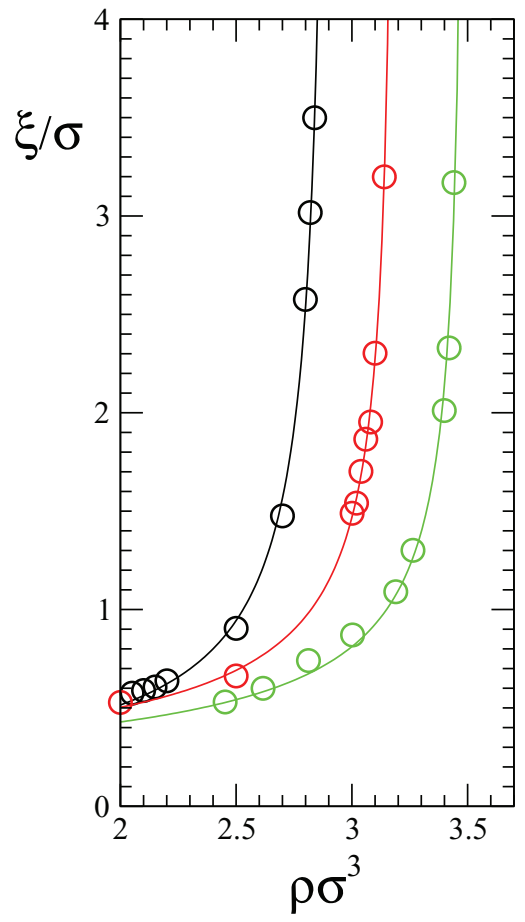

FIG. 5. Value of the orientational correlation length, $\xi$, obtained by fitting the tail of $g_{2}(r)$ to Eq. (6), as a function of density, $\rho$, for hard platelets, $R^{*}$ $\rightarrow \infty$ (black leftward empty circles), hard spherical caps with $R^{*}=2$ (red central empty circles) and hard spherical caps with $R^{*}=8 / 5$ (green rightward empty circles). Curves are fits of the respective data with the function of Eq. (7).

data could be fitted to a function of the form

$$
\xi(\rho)=\xi_{0}\left(\frac{\rho^{*}}{\rho^{*}-\rho}\right)^{v},
$$

with $\xi_{0}$ a length, $\rho^{*}$ the special value at which $\xi$ would diverge, and $v$ an exponent. The optimized values of the pair $\left(\rho^{*}, v\right)$ were: $(2.91 ; 0.77)$ for $R^{*} \rightarrow \infty ;(3.19 ; 0.58)$ for $R^{*}$ $=2 ;(3.49 ; 0.57)$ for $R^{*}=8 / 5$. These data would indicate that, on increasing particle curvature, the exponent tends toward 0.5 , the value predicted by mean-field theory. The numbers in Fig. (7) as well as, a fortiori, their successive elaboration are to be taken with care though. One should take these numbers, especially those in the close neighborhood of the IN phase transition, as a lower bound for $\xi$. One attempt to simulate a system with $N=64000$ hard platelets at $\rho \sigma^{3}=2.84$ and the successive fitting of the $g_{2}(r)$ tail via Eq. (6) led to a value of $\xi=(5.6 \pm 2) \sigma$. More simulations with this and larger number of particles are needed to assess the density dependence of $\xi$ more reliably.

\section{Short-range structure}

What a numerical simulation can probe to a high accuracy is the shape of the positional and orientational correlation functions at short distances. The short-range behavior of these functions provide a very detailed account on the changes that local structure undergoes with density, within a single system and across the various systems, on approaching the IN phase transition. It is useful to start and analyze the behavior of these
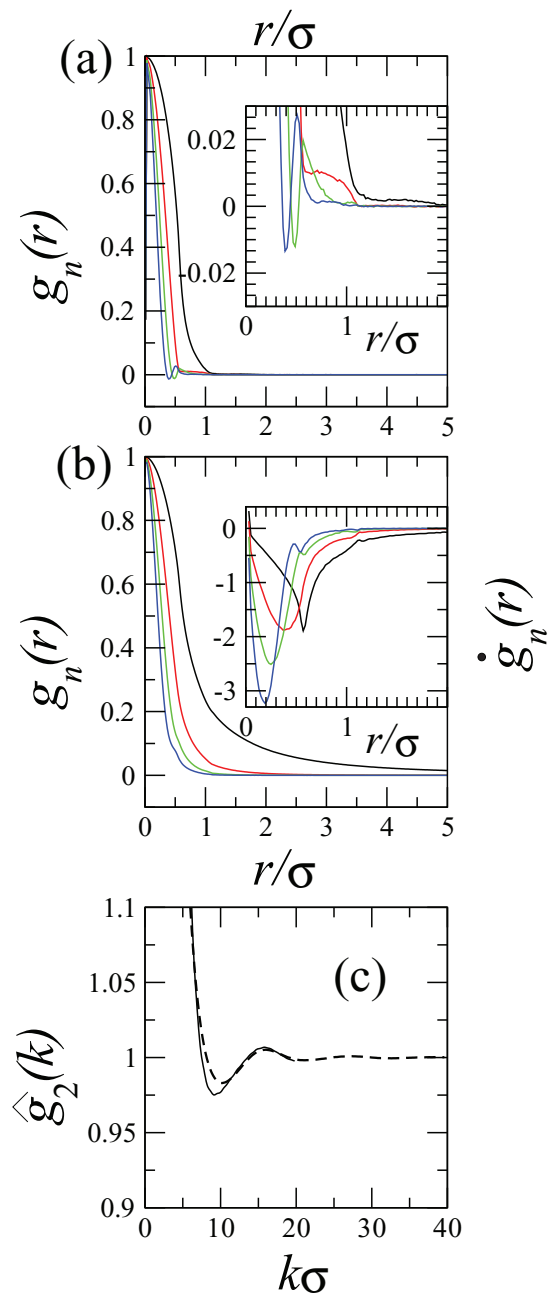

FIG. 6. (a) Orientational correlation functions, $g_{n}(r)$, for a hard platelet system at $\rho \sigma^{3}=1: n=2$ (black line), $n=4$ (red line), $n=6$ (green line), and $n=8$ (blue line). The inset zooms in on the short-range features of $g_{n}(r)$. (b) Orientational correlation functions, $g_{n}(r)$, for a hard platelet system at $\rho \sigma^{3}$ $=2.8: n=2$ (black line), $n=4$ (red line), $n=6$ (green line), and $n=8$ (blue line). The inset zooms in on the short-range features of the derivatives, $\dot{g}_{n}(r)$. (c) The Fourier transform of $g_{2}(r), \hat{g}_{2}(k)$, at $\rho \sigma^{3}=1$ (dashed line), and $\rho \sigma^{3}$ $=2.8$ (solid line).

functions for hard platelets so to have a set of reference results to compare to corresponding results for hard spherical caps. This inter-system comparison informs on the local structural changes that occur in the vicinity of the IN phase transition as hard platelets are progressively curved into lens-like particles.

Fig. 6 shows the functions $g_{n}(r)$ of even rank up to $n=8$, as calculated for a hard platelet system at two densities. One is very low, $\rho \sigma^{3}=1.0$ [Fig. $6(\mathrm{a})$ ]; the other, $\rho \sigma^{3}=2.8$, is very close to the density at which the I phase coexists with a $\mathrm{N}$ phase [Fig. 6(b)]. It is interesting to note that, even at the low density, the $g_{n}(r)$ 's are not featureless [Fig. 6(a)]. Those with $n=2,4$ show a retardation in their decay at distances a bit larger and a bit smaller, respectively, than a platelet diameter, $(2 / \sqrt{\pi}) \sigma \simeq 1.13 \sigma$. The former feature reflects the propensity that two platelets have in arranging edge-to-edge with parallel orientations, while the latter feature is an indication that a pair of platelets can be found in a $\mathrm{T}$-shaped configuration. This fact can also be evinced by the oscillatory behavior that 
$g_{6}(r)$ and $g_{8}(r)$ show at distances comparable to a platelet radius, $r \simeq 0.56 \sigma$. This mild yet non-negligible tendency that two platelets have to arrange perpendicularly is retained at the high density [Fig. 6(b)]. There, it is best appreciated by looking at the derivative of the orientational correlation functions, that, at $r \simeq 0.56 \sigma$, either show a downward cusp, for $n=2$, 4 , or an oscillation, for $n=6,8$. One complementary way to detect this feature is to evaluate the Fourier transform of $g_{2}(r), \hat{g}_{2}(k)$. This function is given in Fig. 6(c) and exhibits a damped oscillation with a period approximately equal to $(2 \pi / 0.56) \sigma^{-1}$. It proved important to jointly consider higherorder rank orientational correlation functions, their derivatives and Fourier transforms. Had the analysis been limited to the orientational correlation functions of low rank, the fine traits in their graph traceable back to a propensity to arrange in a $\mathrm{T}$-shaped configuration even at low density would have remained concealed within the overall decay with distance of these functions.

Their geometry, together with the fact that two of them show a tendency to arrange perpendicularly, make it plausible the presence, within the I phase, of stacks of hard platelets, one stack in turn arranged perpendicularly with respect to another. This feature (that develops further with particle thickness and promotes the formation of a meta-stable cubatic phase in thick hard discs ${ }^{40,41}$ ) leaves its mark also on the shape of pair positional correlation functions.

Fig. 7 shows the layered, $g_{l}\left(r_{\perp}\right)$, and columnar, $g_{c}\left(r_{\|}\right)$, positional correlation functions for hard platelets as a function of density. These functions are defined as follows:

$$
\begin{aligned}
g_{l}\left(r_{\perp}\right)= & \frac{1}{2 \pi r_{\perp} \ell N} \\
& \times\left\langle\frac{1}{\rho} \sum_{i=1}^{N} \sum_{j \neq i}^{N} \delta\left(r_{\perp}-\left|\mathbf{r}_{i j} \times \hat{\mathbf{u}}_{i}\right|\right) \Theta\left(\left|\mathbf{r}_{i j} \cdot \hat{\mathbf{u}}_{i}\right|-\frac{\ell}{2}\right)\right\rangle ; \\
g_{c}\left(r_{\|}\right)= & \frac{1}{\pi \ell^{2} N}\left\langle\frac{1}{\rho} \sum_{i=1}^{N} \sum_{j \neq i}^{N} \delta\left(r_{\|}-\mathbf{r}_{i j} \cdot \hat{\mathbf{n}}\right) \Theta\left(\left|\mathbf{r}_{i j} \times \hat{\mathbf{u}}_{i}\right|-\ell\right)\right\rangle,
\end{aligned}
$$

with $\ell$ taken equal to a hard platelet diameter and $\Theta()$ the usual step-function. The first of these functions gives the conditional probability density to find a particle $j$ at a distance from another particle $i$ equal to $r_{\perp}$, the latter being their distance resolved along a direction perpendicular to $\hat{\mathbf{u}}_{i}$, provided that particle $j$ is constrained within a layer of thickness $\ell$ around particle $i$. The second of these functions gives the conditional probability density to find a particle $j$ at a distance from another particle $i$ equal to $r_{\|}$, the latter being their distance resolved along a direction parallel to $\hat{\mathbf{u}}_{i}$, provided that particle $j$ is constrained within a column of radius $\ell$ around particle $i$. These two functions give complementary pieces of information. Both rapidly reach the limiting value of unity: already at distances slightly larger than a hard platelet diameter particles are positionally uncorrelated. Relevant changes with increasing density occur only in the close neighborhood of a particle. Three are the short-range features exhibited by
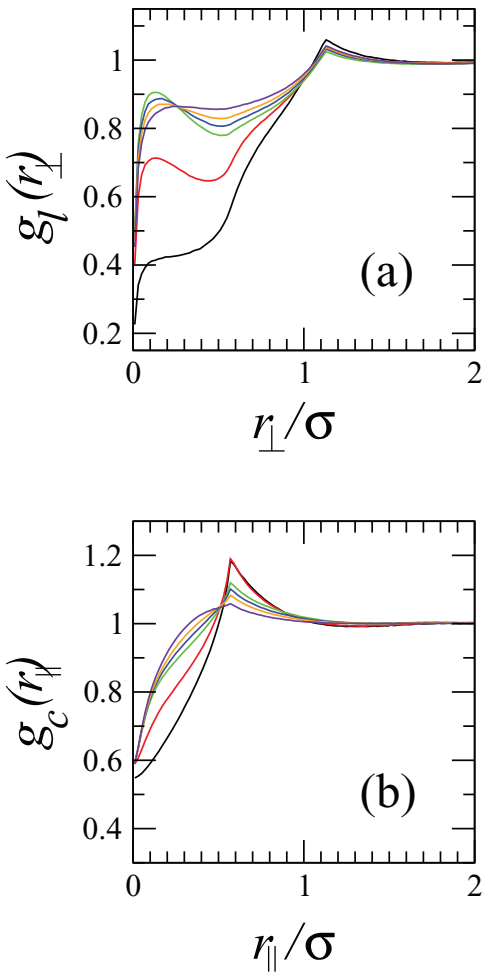

FIG. 7. (a) Layered positional correlation function, $g_{l}\left(r_{\perp}\right)$, for hard platelets at various densities: Looking at the value of $g_{l}\left(r_{\perp}\right)$ at $r \simeq 0.56 \sigma$, from the lowest to the highest: $\rho \sigma^{3}=1$ (black); 2 (red); 2.8 (green); 2.84 (blue); 2.92 (orange); 3 (indigo). (b) Columnar positional correlation function, $g_{c}\left(r_{\|}\right)$, for hard platelets at various densities: looking at the value of $g_{c}\left(r_{\|}\right)$at $r \simeq 0.56 \sigma$, from the lowest to the highest: $\rho \sigma^{3}=1$ (black); 2 (red); 2.8 (green); 2.84 (blue); 2.92 (orange); 3 (indigo).

the $g_{l}\left(r_{\perp}\right)$ 's. Besides the cusp at a distance equal to a platelet diameter, essentially unchanging with density, there is a hump at very short distances and a well at a distance $\simeq 0.56 \sigma$. The cusp at $r \simeq 1.13 \sigma$ reflects the propensity of a pair of hard platelets to arrange parallel edge-to-edge. The increase of density and the approaching to and taking place of the IN phase transition have the effect of making the hump and the well first grow and then level off. Once the $\mathrm{N}$ phase is entered, a rather flat curve has replaced the hump and the well that were present in the graph of these functions at densities within the I phase. The hump at the shortest distances is determined by hard platelets that, as discoid objects, stack on top of one another. Certain of these stacks are in turn arranged perpendicularly. Density exerts its action in promoting the formation of stacks while the tendency of these to arrange perpendicularly is not too much disfavored. It is the occurrence of the $\mathrm{N}$ phase that "cures" their mutual misalignment, that in turn leads to the flattening out of $g_{l}\left(r_{\perp}\right)$ in the $\mathrm{N}$ phase. This is also revealed by the density dependence of the shape of the functions $g_{c}\left(r_{\|}\right)$. On increasing density, one can see that the value that this function takes on at the origin is growing while the cusp-like peak that this function has at $r \simeq 0.56 \sigma$ is decreasing only at the onset of the IN phase transition.

How curving platelets affect these positional and orientational correlation functions is illustrated in Figs. 8 and 9. The first main effect of bending platelets is to promote the decay 

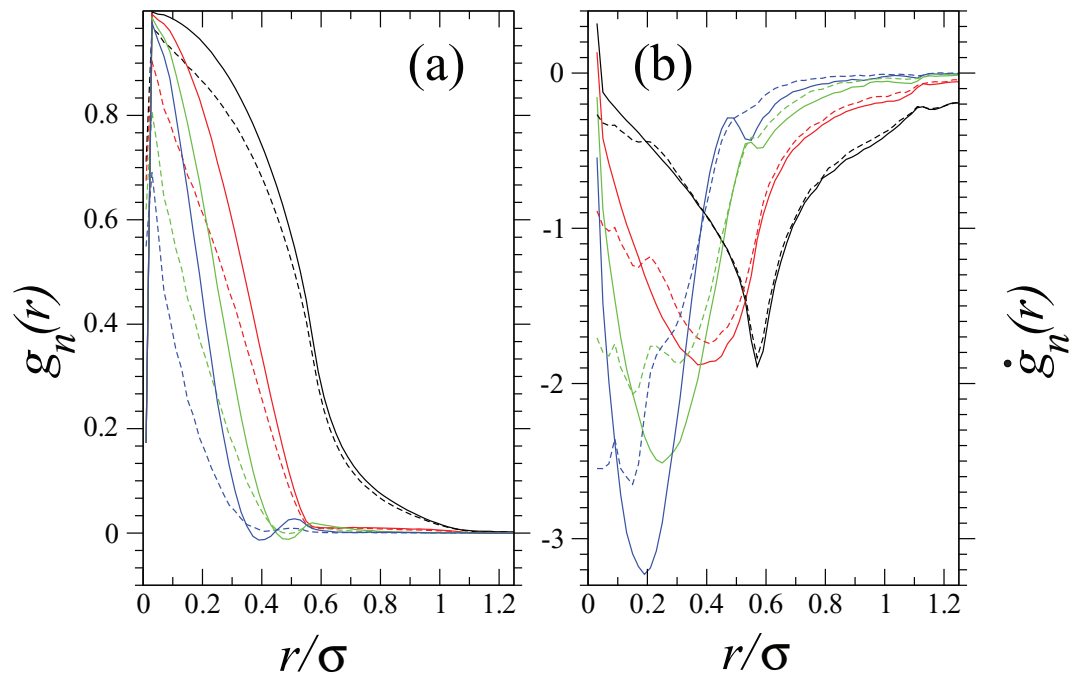

FIG. 8. (a) Orientational correlation functions, $g_{n}(r)$, at $\rho \sigma^{3}=1$ for a hard platelet system (solid curves) and a system of hard spherical caps with $R^{*}=2$ (dashed curves): $n=2$ (black line), $n=4$ (red line), $n=6$ (green line), and $n=8$ (blue line). (b) Derivatives of the orientational correlation functions, $\dot{g}_{n}(r)$, at $\rho \sigma^{3}=2.8$ for a hard platelet system (solid curves) and a system of hard spherical caps with $R^{*}=2$ (dashed curves): $n=2$ (black line), $n=4$ (red line), $n=6$ (green line), and $n=8$ (blue line).

of the orientational correlation functions in the I phase while smoothening out particle propensity to arrange perpendicularly. The oscillation that the higher rank $g_{n}(r)$ exhibited for hard platelets have been damped for hard spherical caps with $R^{*}=2$ [Fig. 8]. Equally smoothen out is the cusp-like peak that the $g_{c}\left(r_{\|}\right)$show at $r_{\|} \simeq 0.56 \sigma$. The second main effect of curvature is to favor stacking of particles on top of one another as the IN phase transition is approached. This is shown by the

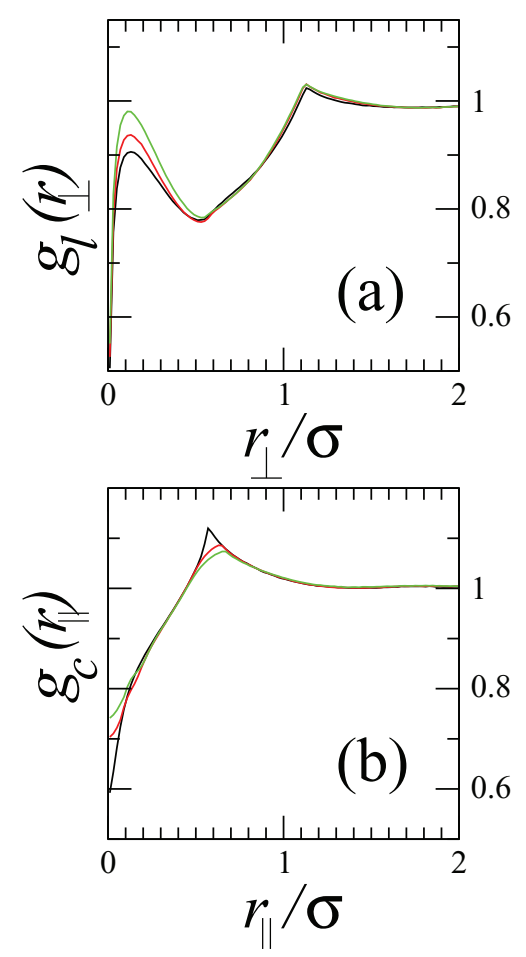

FIG. 9. Layered positional correlation function, $g_{l}\left(r_{\perp}\right)$, in (a), and columnar positional correlation function, $g_{c}\left(r_{\|}\right)$, in (b), for hard spherical caps in the I phase close to the transition to the $\mathrm{N}$ phase: $R^{*} \rightarrow \infty$ at $\rho \sigma^{3}=2.8$ (black curve); $R^{*}=2$ at $\rho \sigma^{3}=3.1$ (red curve); $R^{*}=8 / 5$ at $\rho \sigma^{3}=3.4$ (green curve). increase of the peak of $g_{l}\left(r_{\perp}\right)$ at small values of $r_{\perp}$ and the larger value that $g_{c}\left(r_{\|}\right)$has at the origin. These increases are primarily due to the progressively larger tendency that caps with a larger curvature have to heap up with their unit vectors parallel, in a sterically polar configuration. This is illustrated in Fig. 10 where the first-rank orientational correlation function, $g_{1}(r)$, is shown for systems of hard spherical caps with $R^{*}=2$ and $8 / 5$ at both sides of the IN phase transition. The curves shown corresponding to the $\mathrm{N}$ phase also decay, rather rapidly, to zero, thus indicating that $S_{1}=0$ in this phase too, i.e., the $\mathrm{N}$ phase is apolar. On going from $R^{*}=2$ to $8 / 5$, the value of $g_{1}(0)$ keeps being negative in sign but decreases in modulus, while the successive peak at $r \simeq 0.15 \sigma$ keeps positive and increases. This means that for $R^{*}=8 / 5$ a local parallel arrangement is more favored. Contrary to the antiparallel, sterically antipolar, arrangement, the parallel, sterically polar, arrangement, with caps that tend to slide on the same

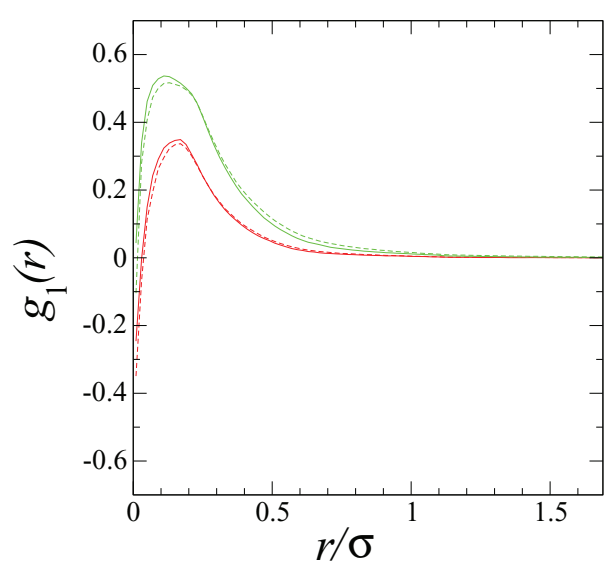

FIG. 10. First-rank orientational correlation function, $g_{1}(r)$, for hard spherical caps with $R^{*}=2$ (red curves) and $R^{*}=8 / 5$ (green curves) in the I (dashed) and $\mathrm{N}$ (solid) phases, at the two sides of the IN phase transition, specifically: for $R^{*}=2$, at $\rho \sigma^{3}=3.10$ and 3.24 ; for $R^{*}=8 / 5$, at $\rho \sigma^{3}=3.44$ and 3.56 . 
spherical surface, is however not compatible with nematic ordering. In fact, the decay of $g_{1}(r)$ is faster in the $\mathrm{N}$ than in I phase.

Thus, hard spherical caps provide an example of geometric frustration as polar ordering, locally favored, cannot propagate globally. This frustration is ultimately responsible for the further weakening of the IN phase transition until the $\mathrm{N}$ is no longer stable: as $R^{*}$ decreases, a system of hard spherical caps find increasingly hard to release the frustration by forming a $\mathrm{N}$ phase, giving up the locally most favorable polar packing for an apolar ordering that can propagate at long range. One in fact knows ${ }^{23}$ that for $R^{*}=3 / 2$ there is no $\mathrm{N}$ phase stable for $N \geq 2000$ but at those densities at which a $N$ phase would be expected the system is in an overall I cluster phase, that allows for an efficient local polar packing at the expense of global nematic ordering. (Such a clustering behavior in lens-like particles, of which the tendency to a mutual perpendicular stacking in hard platelets may be thought of as a forerunner, echos the stacking and clustering behavior observed in smectic ${ }^{42}$ and nematic ${ }^{43}$ phases of bent-core molecular liquid crystals, the molecular "calamitic" analogues of a curved disc.)

\section{CONCLUSIONS}

The overall trend of all the data presented and discussed above is not inconsistent with the existence of a special value of the radius $R^{*}, R_{c}^{*}$, comprised between $8 / 5$ and $3 / 2$, for which $\Delta \rho_{\mathrm{IN}}$ and $\Delta \mathrm{f}$ both vanish; that is, for $R_{c}^{*}$, there is a special value of $\rho, \rho_{c}$, characterized by a diverging orientational correlation length $\xi$. However, nematic order would not develop for $\rho>\rho_{c}$ as it would be superseded by clustering. This is schematized in the upper panels (a) and (b) of Fig. 11. For $R^{*}>R_{c}^{*}$, the situation depicted in Fig. 11(a) takes place. There is a first-order IN phase transition; on increasing density, the $\mathrm{N}$ phase transforms to a cluster I phase, whose equation of state is the high-density continuation of the I phase equation of state at low density. On decreasing $R^{*}$ toward $R_{c}^{*}$, the $\mathrm{N}$ branch moves toward the I-cluster-I branch. For $R^{*}=R_{c}^{*}$, the $\mathrm{N}$ branch meets tangentially the I-clusterI branch at $\rho_{c}$ but for $\rho>\rho_{c}$ it has a larger value of pressure [Fig. 11(b)]. The lower panel (c) of Fig. 11 sketches the phase diagram in the plane $\left(1 / R^{*}, \rho\right)$ and $(\theta, \rho)$ : at the special tricritical point the IN phase transition would be secondorder. (One might draw a parallelism between this phase diagram and those predicted long ago for nearest-neighbor lattice $P_{1}-P_{2}$ models in two ${ }^{44}$ and three ${ }^{45}$ dimensions if one identifies the polar phase predicted there with the I cluster phase here.)

To fully characterize this special tricritical point requires a dedicated study with much larger systems. While waiting for an increase in the computational resources at disposal and an algorithmic improvement to use them most effectively, it would be of interest to carry out numerical simulations on the hard spherical cap's two-dimensional analogues, i.e., hard circular arcs on a plane. Equally of interest would be to conduct experiments on suspensions of colloidal lens-like particles. ${ }^{46}$ It is hoped that this and previous studies ${ }^{22,23}$ will stimulate them.
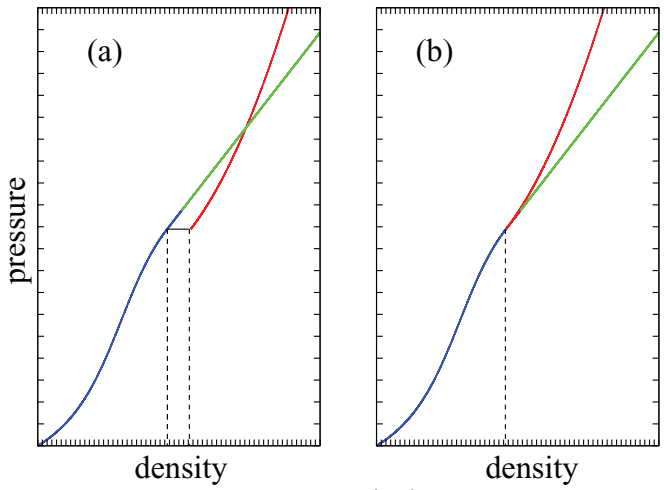

$1 / R^{*}$

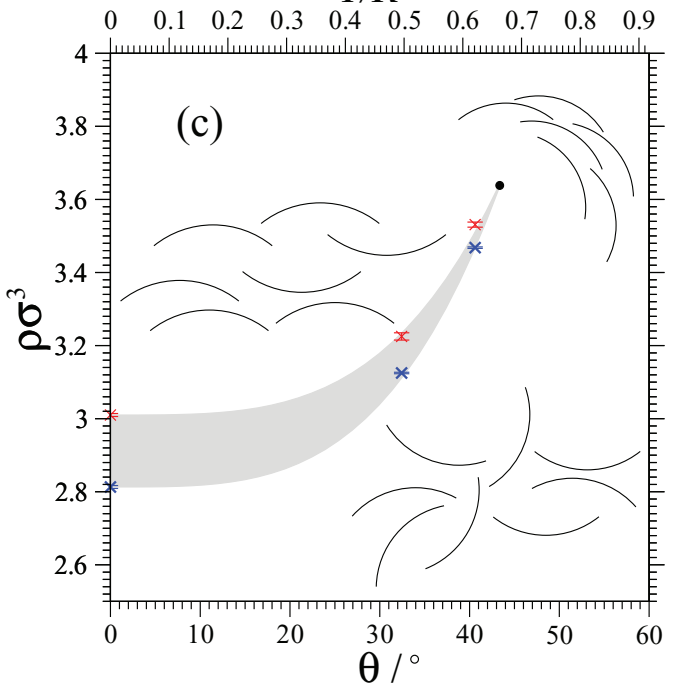

FIG. 11. Upper panels (a) and (b): Schematic equation of state for the case $R^{*}>R_{c}^{*}$ (a) and $R^{*}=R_{c}$ (b); drawn are the I (blue), $\mathrm{N}$ (red), and cluster I (green) equations of state. Lower panel (c): Sketch of the phase diagram in the plane $\left(1 / R^{*}, \rho\right)$ and $(\theta, \rho)$; the blue and red crosses are, respectively, the isotropic and nematic coexistence densities for the three models investigated; the black circle marks the approximate location of the special tricritical point.

\section{ACKNOWLEDGMENTS}

The authors thank D. Bertolini for useful discussions. G.C. is grateful to the Government of Spain for the award of a Ramón y Cajal research fellowship. Italian MIUR PRIN 2008 is acknowledged for the purchase of the computer cluster on which calculations were carried out.

${ }^{1}$ P. G. de Gennes, and J. Prost, The Physics of Liquid Crystals (Clarendon Press, Oxford, 1993).

${ }^{2}$ M. J. Stephen and J. P. Straley, Rev. Mod. Phys. 46, 617 (1974).

${ }^{3}$ S. Singh, Phys. Rep. 324, 107 (2000).

${ }^{4}$ E. F. Gramsbergen, L. Longa, and W. H. de Jeu, Phys. Rep. 135, 195 (1986).

${ }^{5}$ P. K. Mukherjee, J. Phys.: Condens. Matter 10, 9191 (1998).

${ }^{6}$ M. A. Anisimov, Critical Phenomena in Liquids and Liquid Crystals (Gordon and Breach, Philadelphia, 1991).

${ }^{7}$ D. H. Keyes, Phys. Lett. A 67, 132 (1978).

${ }^{8}$ J. F. Li, V. Percec, and C. Rosenblatt, Phys. Rev. E 48, R1 (1993); I. M. Syed, V. Percec, R. G. Petschek, and C. Rosenblatt, ibid. 67, 011704 (2003)

${ }^{9}$ C. M. Care and D. J. Cleaver, Rep. Prog. Phys. 68, 2665 (2005).

${ }^{10}$ J. A. Barker and D. Henderson, Rev. Mod. Phys. 48, 587 (1976).

${ }^{11}$ C. J. Hernandez, and T. G. Mason, J. Phys. Chem. C 111, 4477 (2007); T. J. Merkel, K. P. Herlihy, J. Nunes, R. M. Orgel, J. P. Rolland, and J. M. DeSimone, Langmuir 26, 13086 (2010); S. Sacanna, D. J. Pine, and G. R. Yi, Soft Matter 9, 8096 (2013). 
${ }^{12}$ L. Onsager, Ann. N. Y. Acad. Sci. 51, 627 (1949).

${ }^{13}$ N. Metropolis, A. W. Rosenbluth, M. N. Rosenbluth, A. H. Teller, and E. Teller, J. Chem. Phys. 21, 1087 (1953).

${ }^{14}$ W. W. Wood, J. Chem. Phys. 48, 415 (1968); 52, 729 (1970).

${ }^{15}$ M. P. Allen and D. J. Tildesley, Computer Simulation of Liquids (Clarendon Press, Oxford, 1987).

${ }^{16}$ (a) D. Frenkel, and R. Eppenga, Phys. Rev. Lett. 49, 1089 (1982); (b) R. Eppenga and D. Frenkel, Mol. Phys. 52, 1303 (1984).

${ }^{17}$ M. A. Bates, and D. Frenkel, J. Chem. Phys. 110, 6553 (1999); M. A. Bates, ibid. 111, 1732 (1999).

${ }^{18}$ A. Esztermann, H. Reich, and M. Schmidt, Phys. Rev. E 73, 011409 (2006).

${ }^{19}$ (a) H. Reich, M. Dijkstra, R. van Roij, and M. Schmidt, J. Phys. Chem. B 111, 7825 (2007); (b) H. Reich and M. Schmidt, J. Phys.: Condens. Matter 19, 326103 (2007).

${ }^{20}$ D. L. Cheung, L. Anton, M. P. Allen, A. J. Masters, J. Phillips, and M. Schmidt, Phys. Rev. E 78, 041201 (2008).

${ }^{21}$ P. A. O'Brien, M. P. Allen, D. L. Cheung, M. Dennison, and A. J. Masters, Phys. Rev. E 78, 051705 (2008).

${ }^{22}$ G. Cinacchi and J. S. van Duijneveldt, J. Phys. Chem. Lett. 1, 787 (2010).

${ }^{23}$ G. Cinacchi, J. Chem. Phys. 139, 124908 (2013).

${ }^{24}$ J. F. Sadoc and R. Mosseri, Geometrical Frustration (Cambridge University Press, Cambridge, 1999).

${ }^{25}$ A. T. Gabriel, T. Meyer, and G. Germano, J. Chem. Theory Comput. 4, 468 (2008).

${ }^{26}$ M. He and P. Siders, J. Phys. Chem. 94, 7280 (1990).

${ }^{27}$ M. Matsumoto and T. Nishimura, ACM Trans. Model. Comput. Simul. 8, 3 (1998).

${ }^{28}$ J. Veilliard-Baron, Mol. Phys. 28, 809 (1974).

${ }^{29}$ H. Flyvbjerg and H. G. Petersen, J. Chem. Phys. 91, 461 (1989).

${ }^{30}$ J. E. Mayer and W. W. Wood, J. Chem. Phys. 42, 4268 (1965).

${ }^{31}$ E. P. Bernard and W. Krauth, Phys. Rev. Lett. 107, 155704 (2011); M. Engel, J. Anderson, S. C. Glotzer, M. Isobe, E. P. Bernard, and W. Krauth, Phys. Rev. E 87, 042134 (2013).
${ }^{32}$ Note that in the present work the unit of length is such that it is the platelet's surface area, $\sigma^{2}$, that has a unit value rather than its diameter. The latter is equal to $(2 / \sqrt{\pi}) \sigma$.

${ }^{33}$ (a) R. P. S. Fartaria and M. B. Sweatman, Chem. Phys. Lett. 478, 150 (2009); (b) R. P. S. Fartaria, N. Javid, J. Sefcik, and M. B. Sweatman, J. Colloid Interface Sci. 377, 94 (2012).

${ }^{34}$ D. van der Beek, H. Reich, P. van der Schoot, M. Dijkstra, T. Schilling, R. Vink, M. Schmidt, R. van Roij, and H. Lekkerkerker, Phys. Rev. Lett. 97, 087801 (2006).

${ }^{35}$ M. Simões and D. S. Simeão, Phys. Rev. E 74, 051701 (2006); D. S. Simeão and M. Simões, ibid. 86, 042701 (2012).

${ }^{36}$ I. Haller, Prog. Solid State Chem. 10, 103 (1975).

${ }^{37}$ I. Chirtoc, M. Chirtoc, C. Glorieux, and J. Thoen, Liq. Cryst. 31, 229 (2004).

${ }^{38}$ F. Leenhouts, W. H. de Jeu, and A. J. Dekker, J. Phys. 40, 989 (1979); S. T. Wu and R. J. Cox, J. Appl. Phys. 64, 821 (1988).

${ }^{39}$ R. Berardi, A. P. J. Emerson, and C. Zannoni, J. Chem. Soc. Faraday Trans. 89, 4069 (1993); Y. Olivier, L. Muccioli, and C. Zannoni, ChemPhysChem 15, 1345 (2014).

${ }^{40}$ J. A. C. Veerman and D. Frenkel, Phys. Rev. A 45, 5632 (1992).

${ }^{41}$ P. D. Duncan, M. Dennison, A. J. Masters, and M. R. Wilson, Phys. Rev. E 79, 031702 (2009); P. D. Duncan, A. J. Masters, and M. R. Wilson, ibid. 84, 011702 (2011); M. Marechal, A. Patti, M. Dennison, and M. Dijkstra, Phys. Rev. Lett. 108, 206101 (2012).

${ }^{42}$ P. E. Cladis, H. R. Brand, and H. Pleiner, Liq. Cryst. Today 9, 1 (1999); Ferroelectrics 243, 221 (2000); H. R. Brand, P. E. Cladis, and H. Pleiner, Int. J. Eng. Sci. 38, 1099 (2000).

${ }^{43}$ G. Cinacchi and V. Domenici, Phys. Rev. E 74, 030701(R) (2006); O. Francescangeli and E. T. Samulski, Soft Matter 6, 2413 (2010); O. Francescangeli, F. Vita, C. Ferrero, T. Dingemans, and E. T. Samulski, Soft Matter 7, 895 (2011); C. Zhang, M. Gao, N. Diorio, W. Weissflog, U. Baumeister, S. Sprunt, J. T. Gleeson, and A. Jákli, Phys. Rev. Lett. 109, 107802 (2012).

${ }^{44}$ D. H. Lee and G. Grinstein, Phys. Rev. Lett. 55, 541 (1985).

${ }^{45}$ T. J. Krieger and H. M. James, J. Chem. Phys. 22, 796 (1954).

${ }^{46}$ S. Sacanna, M. Korpics, K. Rodriguez, L. Colón-Meléndez, S. H. Kim, D. J. Pine, and G. R. Yi, Nat. Commun. 4, 1688 (2013). 